\title{
IV. AUTHORS OF PAPERS READ BEFORE THE AMERICAN MATHEMATICAL SOCIETY, 1904-1914
}

Akers, O. P., xII, 225.

Alexander, J. W., II, xIX, 388; xx, 60.

Allardice, R. E., xII, 373, 373; XIII, 99, 369; xIV, 105; xv, 371, 371; xvIr, 445 .

Allen, R. B., XI, 348 .

Altshiller, N., XIx, 498.

Ames, L. D., XI, 56, 348; xIII, 214; xv, 58, 427; xvI, 225.

Archibald, R. C., xx, 509 .

Ashton, C. H., XvI, 293.

Baker, R. P., xv, 426; xvI, 458; xvIII, 433; xIx, 442, 442; xx, 61, 61, 451, 451 .

Batchelder, P. M., xIx, 498, 498.

Bateman, H., XVII, 508; $\mathrm{xIX}, 388,388 ; \mathrm{xx}, 396$.

Bates, W. H., Xv, 295, 427; XVI, 293, 458, 458; xvII, 279; xx, 305.

Bauer, G. N., xIx, 443; xx, 305, 305.

Beal, F. W., XvIIr, 52.

Beal, W. O., XIX, 442.

Beck, H., xvIr, 63.

Beetle, R. D., xx, 286, 396, 508 .

Bell, E. T., XII, 374; XIII, 369; XIX, 164, 164; Xx, 518, 518.

Bennett, A. A., XIX, 164; Xx, 170, 170.

Bennett, E. R., XVII, 223; XVIII, 52; XIX, 219, 219.

Bernstein, B. A., XvIII, 166, 448, 448; XIX, 517; xx, 177.

Bill, E. G., Xv, 277, 278; xvIII, 270, 432; XIx, 498; xx, 508.

Birkhoff, G. D., xIII, 57, 435; XIV, 259, 415; XVI, 55, 283, 395, 395; xVII, 62, 390; XVIII, 53, 53, 486, 486; xIx, 164, 498, 499; XX, 285, 508.

Bjerknes, V. F., XII, 226.

Blichfeldt, H. F., XI, 69, 399, 399; xII, 435, 435; xIIr, 99, 369; xIv, 105, 359 ; XV, 105; XVI, 107; XVII, 115, 445; XVIII, 166, 448; XIX, 517; $\mathrm{xx}, 61$.

Bliss, G. A., XI, 56, 473, 473; XII, 54, 108, 225, 424; XIII, 154, 276, 316, 424; xIV, 249, 352; xv, 58, 278, 294; xvI, 105; xvIII, 53, 160; xIx, 278,$443 ; \mathrm{xx}, 304,304,450$.

Blumberg, H. XIX, 52, 52; Xx, 181, 181, 304, 304, 450.

Bôcher, M., XI, 56; xvII, 279, 507; XIX, 444, 498; XX, 60, 508.

Bolza, O., XII, 326; XIII, 58, 275, 435, 435; Xv, 58; XVI, 458; xx, 59.

Börger, R. L., XIII, 214; XvI, 294.

Bouton, C. L., XI, 348; xvI, 396; xx, 285.

Boutroux, P., Xx, 285.

Bowden, J., XIV, 158; xvII, 390; xvIII, 52, 53.

Bradshaw, J. W., XvII, 62.

Brenke, W. C., XII, 425; XIV, 259; Xv, 207; XVI, 225; xvII, 223; xx, 180.

Bromwich, T. J. I'a., XI, 57.

Brown, E. W., XI, 112, 348; xIv, 249, 249; xvIII, 160.

Buchanan, D., XVII, 507; XIX, 278; Xx, 303, 303 .

Buchanan, H. E., xIv, 259; xv, 295, 295, 426; xvI, 458; xvIr, 507.

Buck, T., $\mathrm{xv}, 426$; xvirI, 448 .

Burgess, H. T., XVI, 55, 458; xVIr, 62, 291, 291, 291; xvIII, 53.

Burns, J. E., Xx, 59.

Bussey, W. H., XI, 473; xII, 54, 326; xvI, 55; xx, 59. 
Cairns, W. De W., xvI, 283.

Cajori, F., XIV, 203; XVI, 170; xvII, 63, 223, 291; xvIII, 52, 433; xIx, 219; $\mathrm{xx}, 60,451$.

Camp, B. H., XviIr, 160, 486; XIX, 388.

Caparo, J. A., XIX, 278.

Carmichael, R. D., XIII, 56, 154, 264, 316, 316, 316, 424, 435, 435, 435 XIV, 54, 54, 54, 157, 157, 352, 352, 416, 416; xv, 157, 157, 277, 277, 419,419 ; XVI, 54; XVII, 62, 168, 508, 508; XVIII, 160, 160, 160, 232, $270,270,433,433$; XIX, 51, 219, 277, 277, 277, 443, 443, 443; xx, 59, 59, 61,450 .

Carpenter, A. F., xvII, 445; xx, 518.

Carstens, R. L., XII, 326.

Carver, W. B., XII, 108; xIII, 57, 264; xIv, 54; xv, 278; xvr, 55, 452.

Castle, E. W., XVIII, 52.

Chambers, G. G., xvI, 54.

Chapman, S., xvIr, 279.

Chessin, A. S., XII, 54; xIII, 214; xIV, 204; XV, 420.

Chittenden, E. W., XvIII, 433; XIX, 443, 443; XX, 451, 451.

Clements, G. R., XvII, 509; xIX, 164.

Coar, H. L., XIr, 280.

Coble, A. B., XI, 232, 233; XIII, 264, 264; XIV, 249; xv, 278; xVI, 55; xvII, $62,279,279$; XVIII, 328; $\mathrm{xx}, 286,508$.

Cole, F. N., XIX, 52.

Colpitts, E. C., XII, 425.

Conner, J. R., xv, 278; xvII, 280; xIx, 278, 278.

Conwell, G. M., Xx, 396.

Coolidge, J. L., XIv, 158; xvI, 283; xvIr, 279; xIx, 278; xx, 285.

Copeland, L. P., xIx, 388.

Craig, C. F., xv, 278; xIx, 498; xx, 61, 396.

Cramblet, W. H., xx, 286.

Crathorne, A. R., XIV, 258; xvII, 509; xIx, 444.

Cummings, L. D., $x x, 508$.

Curtiss, D. R., XI, 56, 56, 462; XII, 225, 436, 436; XIII, 275; XIV, 415; XVI, 293, 458; XVII, 507; XVIII, 432; XIX, 279; xx, 303.

Darwin, G. H., xIv, 158.

Davis, E. W., xIII, 434; xIv, 55, 55, 204; xv, 207, 426; xvI, 225; xvII, 223.

Dean, G. R., XIII, 214; XVI, 225, 293.

Decker, F. F., XVII, 279.

Dederick, L. S., xv, 420; xvI, 55; xrx, 164.

Denton, W. W., xv, 295; xvIII, 433.

Dickson, L. E., XI, 57, 57, 233, 233, 353, 353, 353, 353, 462, 473, 473, 474; XII, 54, 55, 112, 112, 280, 280, 435; XIII, 57, 57, 57, 57, 214, 275, 435, 435; XIV, $54,55,55,106,158,259,259,352,415,415,415$; Xv, 58, 59, 59, 294, 295, 426, 427, 427; XVI, 459; XVII, 62, 62, 62, 62, 291, 291, 509, 509, 509; xvIII, 270, 270, 270; xIX, 278, 278, 443, 443, 443; xx, 115, $304,304,451$.

Dines, L. L., XIv, 415; xvII, 507, 507; xvIII, 160; xIX, 164, 164; xx, 285.

Dodd, E. L., xII, 280, 435, 436; XIII, 214, 435; xvIII, 433; xIx, 52, 52, $220,278,279,443,498 ; \mathrm{xx}, 60,181$.

Dowling, L. W., XIV, 258; Xv, 59.

Dresden, A., xv, 58; xvIII, 270, 270.

Dunkel, O., XviII, 232.

Durand, Wै. F., XvII, 445

Eells, W. C., xx, 177.

Eiesland, J., Xv, 277; xvIr, 63, 63, 390; xvIII, 215; xIx, 278. 
Eisenhart, L. P., xI, 348, 462; xII, 108, 326; xIIr, 57, 424; XV, 420; XVI, 54, 396; XVII, 280, 507; XVIII, 52, 216, 216; XIX, 279, 499; XX, 286, 508.

Epsteen, S., XI, 353, 472; XII, 54; XIII, 214.

Emch, A., XVII, 507; xVIII, 52, 270, 432; xIX, 219, 278; xx, 285, 303.

Ernsberger, I., XVIII, 232.

Escott, E. B., XI, 56; XIII, 435; xIv, 259; xvI, 293, 293.

Ettlinger, H. J., XIX, 498.

Evans, G. C., xvI, 54; xIx, 278; xx, 59, 286.

Field, P., xII, 225; xIII, 435; xvI, 396; xvIr, 62; xvIII, 270; xIx, 278; xx, 304.

Fields, J. C., xvir, 279.

Fine, H. B., Xx, 285.

Finkel, B. F., xIv, 408.

Fischer, C. A., xIX, 388, 499; xx, 396.

Fiske, T. S., XI, 233.

Fite, W. B., XI, 57; xIr, 54; xIII, 56; xIv, 55; xv, 59, 278; xvI, 452; xvIr, 390 ; XVIII, $216 ; \mathrm{xIX}, 280$.

Ford, W. B., XII, 326; XIV, 54, 415; xv, 426, 426; xVI, 55, 458; xVIr, 508.

Forté, H. P., xrv, 408.

Fréchet, M., XI, 57, 233; XII, 54; XIV, 55; XIX, 498; XX, 61.

Frizell, A. B., XII, 225, 225; xv, 207, 208, 294, 295; xvI, 226; xVII, 223, 291, 507; XVIII, 233; XIX, 279; XX, 304, 450 .

Gaba, M. G., xv, 207; XIX, 499.

Galajikian, H., XIx, 278; xx, 170, 286, 396.

Gillespie, D. C., xIII, 265; xIV, 54; xv, 323; xvII, 390.

Glenn, O. E., XI, 348; XIII, 214; XIV, 408; Xv, 58; XVI, 396; xvII, 62, 168, 280,509 ; XVIII, 215; XIX, 53, 444; XX, 60, 285.

Glover, J. W., XII, 280; xv, 294; xvIII, 433.

Graber, M. E., xI, 473; xII, 435.

Granville, W. A., xv, 278.

Graustein, W. C., xx, 286.

Graves, G. H., xx, 170.

Green, G. M., XIX, 388, 499; xx, 170, 170, 170, 286, 396.

Greenhill, A. G., XIv, 56 .

Griffin, F. L., XII, 435; XIII, 264, 424, 424, 424; XIV, 55, 55, 249, 249; $\mathrm{xv}, 58,277$; xvI, 283; xx, 518, 518.

Gronwall, T. H., XVIII, 433, 433; XIX, 52, 53, 53, 220, 220, 220, 220, 279, $279,388,388,388,442,498$; xx, 170, 286, 286, 286, 509.

Grove, C. C., XIII, 435.

Gundelfinger, G. F., XvI, 55, 452.

Halsted, G. B., xIII, 265.

Hancock, H., XIII, 265; XVIr, 390; xIx, 279, 279, 388.

Hardy, J. G., xIv, 249; xv, 278.

Hart, W. L., $\mathrm{xx}, 451$.

Hartwell, G. W., xv, 208.

Haseman, G., XIv, 258.

Haskell, M. W., XII, 112, 112, 374; xIIr, 99; xvIr, 445; xx, 177, 177.

Haskins, C. N., XII, 55, 424; XIV, 259, 352; Xv, 59, 278, 426; XVI, 170; XVIII, 216.

Hathaway, A. S., xIv, 259.

Hawkes, H. E., XIII, 265; XIv, 249.

Hawkesworth, A. S., xvI, 54; xvII, 279.

Hazlett, O., Xx, 303.

H'Doubler, F. T., Xx, 59. 
Hedrick, E. R., XI, 57, 57; xII, 424, 424, 435; xII, 316, 435; xIV, 54, 54, 204, 259; XV, 59, 208; XVI, 225; XVII, 224; XVII, 232, 233; XIX, 220; $\mathrm{Xx}, 59,180,181,450$.

Hennel, C. B., XIX, 279.

Hewes, L. I., XIv, 249; Xv, 59.

Hildebrandt, T. H., xv, 427; xvII, 433; xx, 450.

Hill, G. W., xIv, 352.

Hiltebeitel, A. M., xI, 348.

Hodge, F. H., xIV, 415 .

Hodgson, J. E., XVIII, 328.

Hoskins, L. M., xI, 69; XIII, 99, 369; xIv, 359, 359; xvr, 107, 400; xx, 177.

Howland, L. A., xvIII, 215, 486; xx, 286.

Huntington, E. V., XI, 57, 233, 233; XII, 55, 225; XIV, 408, 408; XVI, 284; XVIII, 160; XIX, 164, 279, 499; Xx, 285, 396.

Hurwitz, W. A., XVI, 55; xvIII, 52, 52; XIX, 277, 278, 499; xx, 285.

Hutchinson, J. I., XII, 54, 225; XIII, 57, 264; XIV, 249, 408; XV, 277.

Ingold, L., XI, 57; XII, 435; XIII, 435, 435; xIV, 203, 204; xV, 58; XVI, 226; XVII, 63, 223, 223; XVIII, 232, 270; XIX, 220; Xx, 180.

Irwin, F., XIV, 352; xV, 158.

Jackson, D., xvI, 54; xvIII, 328, 328, 486; xIx, 52, 164, 388; xx, 60, 285, 396.

Jackson, W. H., xv, 420, 420; XvI, 283.

James, G. O., xIV, 203; xvI, 225; xvIII, 233, 432.

Joffe, S. A., XvIII, 328; Xx, 508.

Karpinski, L. C., xvI, 283; xvIr, 292, 292, 508; xvIIr, 52, 433; xIx, 52, 279, 279, 442, 442; xx, 59, 303.

Kasner, E., XI, 112, 233, 462, 462; XII, 54, 108, 225, 424, 424; XIII, 57, 57, $154,265,265,425,425$; xIv $55,55,158,352,352,408 ; \mathrm{xv}, 59,157,278$, 278 , 323, 420; xvI, 54, 54, 105, 170, 284, 284, 396, 452; xvII, 63, 168, $390,390,507$; XVIII, 160, 215; XIX, 164, 279, 388, 499; xx, 60, 170, 285.

Kellogg, O. D., xII, 436; xIII, 56, 214, 276; xIv, 204, 416; xvII, 224; XVIII, 232; xIx, 444; xx, 181.

Kells, L. M., xx, 286.

Kempner, A. J., Xx, 181.

Kenyon, A. M., xx, 451 .

Keyser, C. J., XII, 225, 326, 424; xIII, 264, 264; xv, 278; xvI, 284; xvII, 507 ; XIX, 279.

Killam, S. D., XIX, 499.

Kircher, E., Xx, 450.

Kuschke, C., xvII, 445.

Lambert, P. A., xIV, 408; xvr, 170.

Lamond, J. K., xvII, 486; xx, 286, 508.

Landau, E., XVIII, 52.

Landry, A. E., Xv, 158.

Laves, K., XII, 280; XIII, 435; xvIr, 292; xvIII, 432; xIx, 444; xx, 61.

Lefschetz, S., xvIII, 52, 53, 232, 328, 432, 448, 486; xIx, 52, 220, 443; xx, 180.

Lehmer, D. N., XI, 69; xII, 373, 373; xIV, 105, 105, 359; xvI, 107, 400; XVIr, 115, 445; xIX, 174.

Leib, D. D., Xv, 278.

Lennes, N. J., xI, 353, 353; XII, 54, 280, 280, 280, 435; XVII, 389, 389, 390, 509,509 ; xVIII, 160, 486, 486; xIx, 388, 388 . 
Leonard, H. B., XIII, 57.

Leuschner, A. O., XI, 399; XII, 112; XVI, 107; XVIII, 166.

Levi, B., XIII, 154.

Lewis, C. I., XIX, 174.

Lipka, J., xIv, 359; xv, 277; xvI, 54; xvIr, 280; xIx, 279.

Loewy, A., XI, 473.

Longley, W. R., XII, 435; xIII, 264; xV, 59, 277; xvI, 55; xvIr, 279; XVIII, $486 ; \mathrm{xx}, 286$.

Love, C. E., XIx, 280, 443; xx, 304.

Lovett, E. O., XI, 462; XIV, 54, 54, 249, 249; xv, 277; XvII, 63.

Lovitt, W. V., xvIr, 445.

Lunn, A. C., XII, 280; XIII, 276; XIV, 415, 415, 415; Xv, 294, 295, 295; XVI, 293, 293; XIX, 443, 443.

Lytle, E. B., XIv, 408; xx, 59.

McClenon, R. B., XI, 462.

McDonald, J. H., XII, 373, 374, 374; xIv, 105, 105, 359; xv, 105; xvIr, 445.

McDonnell, J., XIX, 443.

McEwen, G. F., xv, 371; xvI, 107.

McKelvey, J. V., xvi, 283.

McKinney, T. E., XIII, 265, 425, 425; xvI, 54; xx, 450.

McMahon, J., XIII, 57, 264; XIv, 55.

Macaulay, F. S., XI, 57.

MacDonald, W. E., xvir, 291.

MacGregor, H. H., XvI, 293.

Maclay, J., XvII, 509.

MacMahon, P. A., XI, 69.

MacMillan, W. D., XII, 435; XIv, 416; Xv, 427, 427; XvIr, 291, 507, 507, 509 ; XIX, 280, 280; XX, 303, 303, 451, 451.

MacNeish, H. F., XVI, 284; xvII, 291.

Manning, W. A., XI, 399; XII, 54, 112, 373; xIII, 57, 99, 369; xv, 371; XVI, 294, 400; XVIII, 166; XX, 177.

March, H. W., Xx, 61.

Marriott, R. W., XvII, 509.

Marshall, E. R., xvIII, 486.

Marshall, W., xvIr, 508; xx, 59.

Maschke, H., XI, 473, 473; XII, 436.

Mason, M., XI, 112, 232, 462; XII, 54, 108, 226, 424; XIII, 57, 57, 73, 154, 264 ; XIV, 249, 352; Xv, 58.

Mason, T. E., Xx, 59, 450, 450, 451.

Miles, E. J., Xv, 294, 294; xvII, 279, 389; XvIII, 328; xx, 509.

Miller, G. A., XI, 57, 69, 112, 399; XII, 54, 108, 112, 280, 374, 374, 424; XIII, 57, 154, 154, 214, 265, 275, 316, 369, 424, 425, 435; XIV, 54, 54, $55,105,204,258,352,359,415$; xv, 58, 59, 157, 207, 277, 426 ; xvI, 54, $170,283,294,396,452,458,459$; XVII, 62, 62, 168, 168, 223, 291, 389, 508 ; XVIII, 52, 52, 166, 215, 270, 328, 433; xIX, 52, 278, 442; xx, 177, $304,304,304,450$.

Miller, K. G., XIv, 55.

Miser, W. L., XIX, 442.

Mitchell, B. E., xx, 396.

Mitchell, H. H., XVI, 283, 452, 452; xVII, 63, 168, 279; xvIII, 160, 328; $\mathrm{XIX}, 52,499 ; \mathrm{xx}, 508$.

Mitchell, U. G., XVII, 508.

Montgomery, W. J., xvII, 508.

Moore, C. L. E., XII, 225; xv, 277; xvI, 452; xvIr, 279; xvIII, 215, 215, 486,486 ; xx, 508, 508.

Moore, C. N., xIII, 316; xIv, 408; xv, 59; xvI, 170, 293; xvII, 390, 509; XVIII, 215 ; $\mathrm{xx}, 451$. 
Moore, E. H., XI, 233; XII, 280; XIII, 73, 214, 214; xIV, 259; xv, 295; XVIII, 433; XIX, 278, 444; Xx, 60 .

Moore, R. L., XI, 472, 473; XIV, 158; xvI, 459; xvIr, 507; xvIII, 215, 486; $\mathrm{XIX}, 498 ; \mathrm{XX}, 509$.

Morehead, J. C., XI, 112, 462; XII, 280, 435; XIV, 258; XV, 420, 426, 426.

Moreno, H. C., $\mathrm{Xv}, 105$.

Morgan, F. M., XIX, 52, 278, 499.

Moritz, R. E., XI, 399; XII, 112; XIII, 99; XVII, 445; Xx, 518, 519.

Morley, F., XI, 233; xII, 54; xIII, 57; xIv, 249; xv, 277.

Morris, R., XII, 54.

Morrison, F. M., Xx, 518 .

Moulton, E. J., XIv, 415; xx, 304.

Moulton, F. R., XII, 55, 435; XIII, 58; XIV, 259; Xv, 427, 427; XVI, 294, 459; XVII, 292, 509, 509; XVIII, 270, 433; XIX, 443; Xx, 304, 451.

Neikirk, L. I., xIII, 434; xv, 427; xvI, 294, 458; xvII, 292, 509; xIX, 517; $\mathrm{xx}, 518$

Newson, H. B., XII, 280; XIII, 214; XIv, 204; Xv, 59; XVI, 55, 225.

Noble, C. A., XII, 112; xIV, 105; Xv, 105; XVI, 400.

Nyberg, J. A., xvII, 509.

Osgood, W. F., XIII, 424; xIV, 203; xv, 420; xvIII, 328; xIx, 164; xx, $60,60,115,286$.

Owens, F. W., XI, 473.

Peirce, G., XII, 226.

Pell, A., XIII, 214.

Pell, A. J., xv, 427; xvI, 54, 293, 458; xvII, 63; xIX, 52; xx, 508.

Phillips, H. B., Xv, 278; XVI, 452, 452; xvII, 280; xvIII, 215, 486, 486; $\mathrm{XIX}, 279$.

Pierpont, J., XI, 348, 462, 462; XII, 108, 424; XIII, 264.

Pitcher, A. D., xv, 427; xvII, 223; xvIII, 486; xIx, 443, 499, 499; xx, 451

Poincaré, H., XI, 57.

Ponzer, E. W., xvI, 400; xvII, 445.

Poor, V. C., $\mathrm{xx}, 303$.

Porter, M. B., XI, 462, 462; XIII, 214, 214, 214; xVI, 225.

Powers, R. E., XVIII, 160.

Pupin, M. I., XII, 226.

Putnam, T. M., XI, 69; xII, 374; xIX, 517.

Quinn, J. J., xIr, 226.

Rabinovitch, I. E., XII, 425.

Ragsdale, V., xI, 462.

Ranum, A., XII, 112, 373; XIII, 56, 154, 264; XIV, 55, 249; XV, 58, 58, 105, $278,278,426$; XVI, 283, 284, 396; XVII, 62, 62, 292; xVIII, 52, 215; XIX, 52,279

Reaves, S. W., XIX, 219.

Reddick, H. W., xVI, 55; xIx, 164; xx, 286.

Reed, F. W., XVI, 54 .

Richardson, R. G. D., xII, 225, 424; xIII, 56, 316, 424; xIv, 249; XVI, 55, $55,283,452$; XVII, 279, 508; XVIII, 215; xIX, 388, 499; xx, 59.

Richardson, S. F., XIII, 154.

Rider, P. R., Xx, 509 .

Rietz, H. L., XI, 473; xII, 435; xv, 427.

Risley, W. J., xvII, 291.

Robinson, L. B., xIX, 52, 388; xx, 285.

Roe, E. D., Xv, 420; XVII, 63, 63. 
Roever, W. H., XII, 424, 424; XIV, 352; xv, 207; xVI, 225; xvII, 223, 509; XVIII, 232, 432, 432, 432; XIX, 219; xx, 171, 396.

Root, R. E., XVII, 508; XVIII, 270; XIX, 442.

Rowe, J. E., XVII, 62, 508; xVIII, 52, 215, 215, 328; xIx, 52, 278, 498; xx, $60,60,508$.

Royce, J., XI, 462.

Runge, C., xvi, 170.

Running, T. R., xx, 61.

Safford, F. H., Xvr, 452; XvIr, 509; xIX, 52.

Sanderson, M. L., XIX, $442 ; \mathrm{Xx}, 61$.

Saurel, P. L., XIII, 425; xIv, 408; Xv, 420; xvII, 279.

Schmiedel, O., xIII, 214.

Schottenfels, I. M., XI, 57, 462, 462; XII, 326; XIII, 424; XVI, 459; XIX, 279,443 .

Schultze, A., XI, 461.

Schur, I., XIv, 416.

Schwatt, I. J., xvI, 55.

Schweitzer, A. R., XII, 435; XIII, 56, 56, 275, 434; XIV, 55, 259, 415, 415, 416; Xv, 295; xVI, 55, 294, 294; xvII, 508, 508; XvIII, 160, 486, 486; $\mathrm{xIx}, 444,444 ; \mathrm{xx}, 60,60,60,60,181,303,304,304,396,396,450$, $451,451,508$.

Scott, C. A., XI, 348; xIII, 154.

See, T. J. J., XI, 399 .

Sharpe, F. R., xI, 348; xIII, 56, 265; xIv, 352, 352; Xv, 58, 277; xvI, 54; XVIII, 215; XIX, 52, 52, 52, 278; Xx, 60, 61, 396, 396.

Shaw, J. B., XI, 57, 473, 473; XII, 280, 435, 435; XIII, 275, 275; XIV, 204, 258; Xv, 58, 295; xVI, 225, 294, 458; XVII, 292, 508; XVIII, 270; xIx, $279,279,443$; $\mathrm{xx}, 60,304$.

Sheffer, H. M., xvI, 283; XIx, 278, 498; xx, 61, 61.

Siceloff, L. P., XVIII, 160, 486 .

Silverman, L. L., xVII, 168; xVIII, 216, 216; xIx, 280.

Sinclair, M. E., XIV, 55; xv, 278.

Sisam, C. H., XI, 233; XII, 54; XIII, 57, 275, 435; XIV, 55, 258, 258, 415; XVI, 294, 458; XVII, 508 .

Slepian, J., XIX, 498.

Slobin, H. L., XVII, 292; xIx, 443; xx, 305.

Slocum, S. E., XIII, 264; XIV, 408; XVII, 62; xvIII, 215.

Smail, L. L., XVII, 446; XIx, 280, 498; XX, 518, 518.

Smith, A. W., XI, 348.

Smith, B., XI, 233, 353; XII, 108.

Smith, C. E., XII, 226, 326.

Smith, P. F., XVI, 55, 395.

Smith, W. M., xvIII, 215; xx, 518.

Snyder, V., XI, 112, 232; XII, 55, 326, 424; XIII, 57, 264; XIV, 54, 55, 352; $\mathrm{xV}, 58,58,278,323$; xvI, 54, 452; xvII, 62, 279; xvIII, 53; xIx, 280; $\mathrm{xx}, 285,396$.

Spunar, V. M., xvirr, 433.

Stäckel, P., XII, 326 .

Stager, H. W., xv, 371; xvIII, 166; xIx, 517.

Stephens, R. P., xII, 226; xIII, 265, 316; xIV, 55.

Stickelberger, L., xIII, 56 .

Story, W. E., xIII, 264.

Stouffer, E. B., xVII, 509; xvIII, 433; xx, 451.

Stringham, I., XI, 399.

Stromquist, C. E., XIIr, 424.

Study, E., Xv, 58; xvI, 396.

Sullivan, C. T., xIx, 499; xx, 304. 
Suter, J. D., xIv, 359.

Swift, E., XIV, 408.

Taber, H., XI, 112.

Tappan, H., Xx, 285.

Taylor, E. H., XvI, 452; xIX, 164.

Townsend, E. J., XII, 55; xv, 59.

Tracey, J. I., Xx, 286.

Underhill, A. L., XI, 473; xIII, 424; xIv, 259; xv, 295; xx, 304.

Urner, S. E., XVIII, 328.

Van Benschoten, A. L., xIV, 55; xvIII, 215.

Van der Vries, J. N., Xv, 207.

Vandiver, H. S., XI, 57; XVIII, 270; XIX, 499; xx, 395, 508.

Van Vleck, E. B., XI, 112, 462; XIII, 435; XIV, 352; XVI, 294, 294; XvII, 508,$508 ; \mathrm{xx}, 59$.

Veblen, O., XI, 57, 353, 473, 473, 473, 474; XII, 326; XIII, 264; XIV, 55, 157,249 ; XVI, 55; XVIII, 53, 486; XIX, 52, 388.

Volterra, V., XIX, 164.

Wahlin, G. E., xII, 424; xvI, 293; xvII, 508; xvIII, 432; xx, 305, 450.

Wear, L. E., Xx, 518.

Webster, A. G., XII, 226; XvIIr, 53, 53, 53.

Wedderburn, J. H. M., XI, 353, 473, 473; xv, 59; xx, 286.

Weeks, E., xx, 181.

Wernicke, A. L. P., XIII, 214; xIv, 203, 203; xv, 207, 208; xvI, 225.

Western, A. E., xv, 426.

Westfall, W. D. A., XII, 436; XIII, 214; XVI, 225; xx, 59, 181, 450.

Westlund, J., XII, 280; XIV, 415; XVI, 396; XVII, 62, 291; XIX, 278.

White, H. S., xI, 57, 353; xII, 55, 108; xIv, 55; xv, 277; xIX, 52.

White, M. B., XvIII, 232.

Whitney, A. W., xI, 399; xv, 371; xvIII, 166; xIx, 174.

Whittemore, J. K., xviII, 432.

Wilczynski, E. J., XI, 69, 399, 462; xII, 112, 373; XIII, 73, 369; XIV, 258, 415 ; $\mathrm{xV}, 59,294,427$; xVI, 293, 458; xVII, 291; xVIII, 270, 432; xIx, $53,279,443 ; \mathrm{xx}, 60,304,451$.

Wilder, C. E., XIX, 388; xx, 509.

Williams, F. B., xvII, 292.

Williams, K. P., xVIII, 328; xIx, 443, 499; xx, 304.

Wilson, A. H., XI, 353 .

Wilson, E. B., XI, 462; XII, 326; XIII, 57, 57, 58, 265, 316; XIV, 249, 249 , 408,408 ; $\mathrm{xv}, 59$, 158; $\mathrm{xvIII}, 215$; $\mathrm{xx}, 396$.

Wilson, N. R., xI, 473.

Wilson, R. E., XII, 436, 436.

Winger, R. M., XIX, 280; Xx, 61, 61, 519.

Wright, J. E., XI, 233, 462, 462; XII, 226, 226; XIII, 424; xv, 420.

Young, A. E., XI, 353; XII, 280; XIII, 435; XIv, 415; Xv, 426; XvII, 507.

Young, J. W., XI, 57; XII, 280, 424; XIII, 57; XIV, 249, 352, 352, 408; XV 59 , 295; XVI, 294; XVII, 224; XVIII, 216, 216; XIX, 499, 499. 